\title{
Optimization of Mobile Ad Hoc Network DSDV and OLSR Using Evolutionary Algorithm for Elearning induction Model
}

\author{
Fauzan Prasetyo a, , Moh. Nazir Arifin ${ }^{\text {b*, Agus Irmawan c }}$ \\ ${ }^{a, b}$ Informatic Engineering Departement, Madura University \\ Panglegur, Tlanakan, Pamekasan, East Java, Indonesia \\ ${ }^{b}$ Civil Engineering Departement, Madura University \\ Panglegur, Tlanakan, Pamekasan, East Java, Indonesia \\ *Corresponding author's e-mail: nazirarifin@gmail.com
}

\begin{abstract}
The e-learning induction model that is well informed by the theory and practice is a sure way of being responsive to the dynamism of educational technologies. Common problem that must be taken as consideration and must be resolved in urban areas and the organization is an efficient message delivery in (MANET) Mobile Ad hoc Network. To get good and efficient communication, an algorithm must pay attention to several aspects such as the density of neighbouring node, shape and network size, channel priority level and used of message. Some previous studies attempted to propose solutions for delivering messages, but finding the optimal problem solution that will be use is very difficult. In our research, we sugested an optimization on MANET by using an EA. The algorithm will provide several solutions to the problem of sending messages to MANET. Our goal is able to determine the optimal communication strategy for each node in network. By using (EA) evolutionary algorithm in (n-2) network simulator, we found that result is promising for message delivery optimization to destination for using in system Elearning model network.
\end{abstract}

Keywords: MANET; evolutionary algorithm; message delivery optimization

\section{Introduction}

As the increasing of infomation technology development, E-Learning induction requires a commitment to lifetime learning for purpose of applying the oppurtunities of digital network and media, certain types of special networks which are needed by people and not depending on infrastucture are highly required. This infrastructure dependency can be reduced by utilizing 
Mobile Ad Hoc Network (MANET). MANET is a collection of nodes that move dynamically in order to form a temporary network without using an existing network structure and each node can be a laptop, cellphone, or other device that has wireless mobile device (1). Every wireless mobile device on MANET will perform task normally carried out by network infrastructure such as packet relay, route searching, network monitoring, communication security and so on.

Broadcasting is used by MANET as one of the basis for sending data packet and routing in MANET network does not have subnet work so it does not have an explicit router because each node can be a router for all of its members. Data packet are sent from one node to another by broadcasting it until reach the destination node. Sending data packet effectively in MANET which has high mobility and large areas (for example on VANET) is a difficult task to achieve. An efficient algorithm must take account several aspects such as density of environment, size dan shape of the network.

Many routing protocols have been developed for ad hoc network and they can be categorized into three types: reactive, proactive, and hybrid. In reactive protocols such as Distance-Sequenced Distance Vector Routing (DSDV) (2) and Ad hoc On-demand Distance Vector Routing (AODV) before doing communication between nodes, the initial node will request the destination route of data packet by spreading it to all destination route node and expect a response from destination node. Conversely, the proactive protocol such as Optimized Link State Routing (OLSR) always update the routing information continueously in order to get a fixed map or vision on network topology they used. Hybrid protocol is the another improvement of proactive dan reactive protocols where this protocol has both proactive and reactive protocols in once such as TORA and ZRP (3).

Multipath protocols routing that are used by Minimum Delay Metric OLSR is a protocols that was developed from OLSR (4), which also added Djikstra Algorithm to obtain flexibility in selecting route from data transmission.

The purpose of this research is to determine the optimal communication strategy for each node based on density, which is expected to reduce problems in packet delivery that often occurs. This paper uses network simulator (ns-2) and proposes the using of Evolutionary Algorithm (EA). By utilizing the proposed algorithm can provide optimal solution on relay problems that are often encountered such as packet delay and over head packet. 


\section{Research Methodology}

a. OLSR
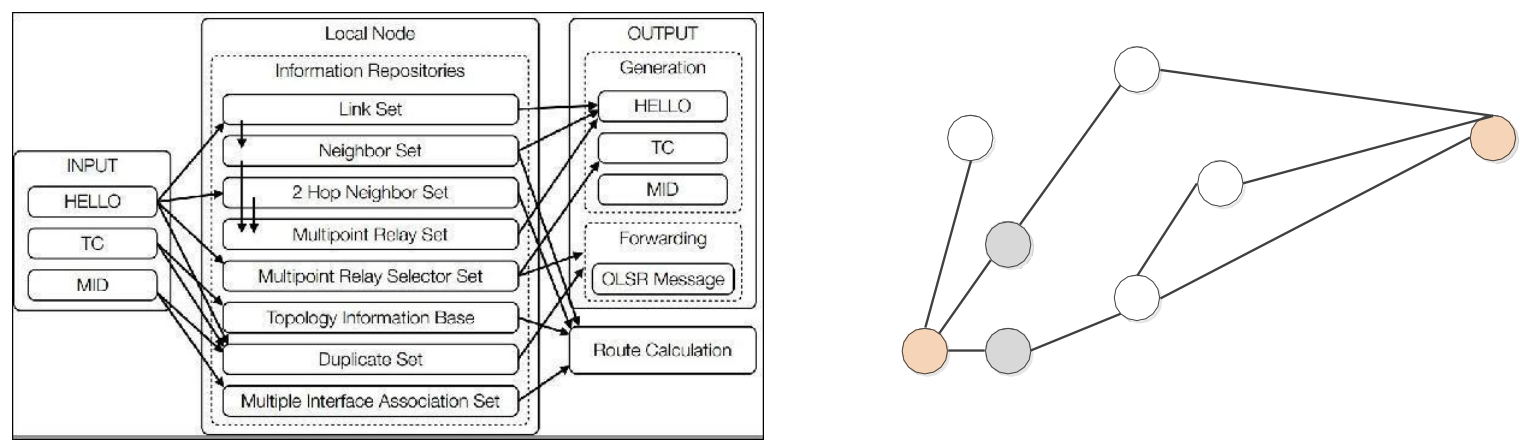

Figure 1. OLSR (Jasquest, dkk, 2001)

OLSR uses 3 ways to control messages: HELLO, Topology Control (TC), and Multiple Interface Declaration (MID), Hello messages sent periodically to all neighboring nodes. These Hello messages contain information about neighboring nodes, node that has been selected as MPRs and has list of neighbors.

OLSR message Topology Control (TC) with MPR mechanism (Multipoint relying) to spread neighbor information across the network. This message contains all nodes selected to be MPRs. MID messages are used to notify that the node is running on OLSR by using more than one interface. This MID message fills all network formed by MPRs.

After getting information about the neighbor node, the neigbor node is selected as MPR (Multi Point Relay), where only the MPR node can forward the broadcast message. The closest path is chosen to send the message

b. DSDV

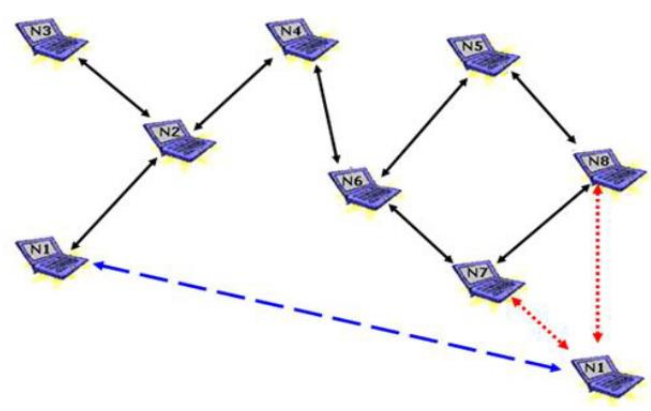

Figure 2. DSDV

Destination Sequenced Distance Vector (DSDV) is a hop-by-hop vector routing protocol that requires information on each node to periodically make updates to the routing table. The tabledriven algorithm is based on modifications from Bellman-ford routing mechanism. Each node in the network has a routing table for each destination in the network and the number of hops needed to reach the destination. This mechanism allows the protocol to avoid forming the same routing loop. 
c. Proposed method using Evolutionary Algorithm

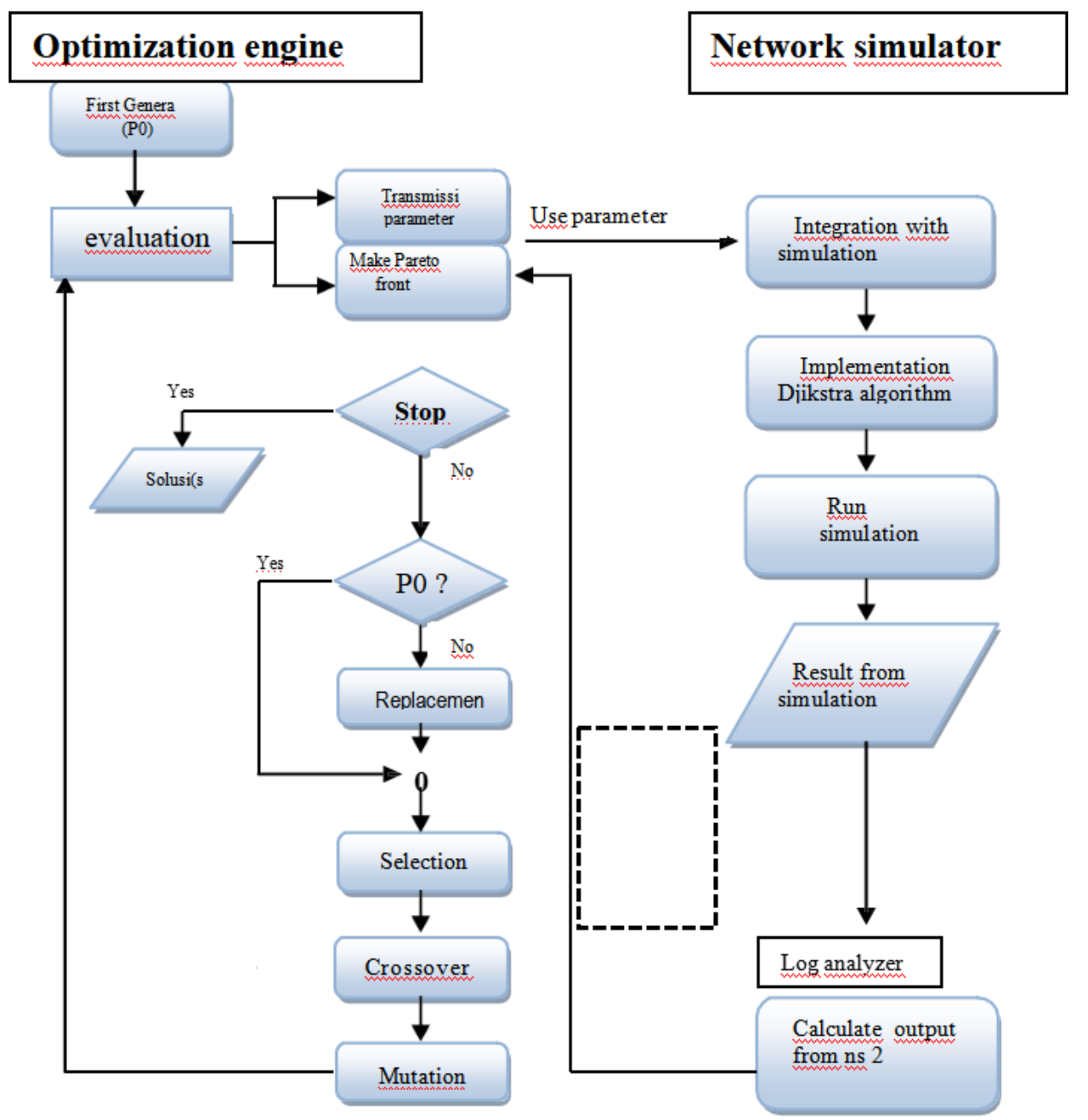

Figure 3. Subsystem interaction

d. Determination and selection of NS-2.35 scenario in the Evolutionary Algorithm

The proposed method is based on three main modules: optimization engine, network simulator and $\log$ analyzer. The three sub-systems work together to provide optimal solutions to the problem that commonly occurs in MANET network in OLSR protocol.

In optimization engine, the process starts from making n-individuals (as many as 5 or 10 individuals) where the individuals must comply with the specified parameters namely $\mathrm{Pr}, \mathrm{Nr}, \mathrm{Dr}$ and TTL where each individual will be measured to get fitness value. After knowing the fitness value from each individual, two best fitness values are chosen to be parents.

After obtaining parents. The value of one parent will be entered in the network simulator (ns2) where the value of the 4 parameters will be processed and produce simulation logs which is in ns-2 known as data trace (.tr) and node simulation (.nam). The result of simulation log will be entered in the "log analyzer" where the results of the $\log$ simulation will be taken 4 values namely $\mathrm{Pl}, \mathrm{Ed}, \mathrm{Pd}$ and FR. Those values obtained from simulation logs is search "pareto front" as equation below: 


$$
P F *:=\{u=F(x) \mid x \in P *\}
$$

After getting the value of "pareto front" then the fitness value of the n-individuals that we have obtained is "parents" where fitness value obtained from following equation:

$$
\text { fitness }=\min \quad \text { wifi } x
$$

The fitness values obtained will be compared with the value of the pareto front. If the fitness value meets or matches the pareto front then the value can be taken as solution. But if the value does not meet the value of the pareto front, a process in figure 2 above will be performed, by first checking the value of population (Po) wether there is a value of population. If there is no then will be done "replacement" where the value entered is the value of the initial population before the simulation process (parents). If there is already a value yes then the next process "selection" process will be carried out by selecting parent 1 and parent 2 . After finding the values of the two parents, then it will be converted into binary form followed by "selection" process. Next process is do combination process namely "crossover and mutation" in which the process is carried by crossovering the binary value of parent 1 and parent 2 as show below:

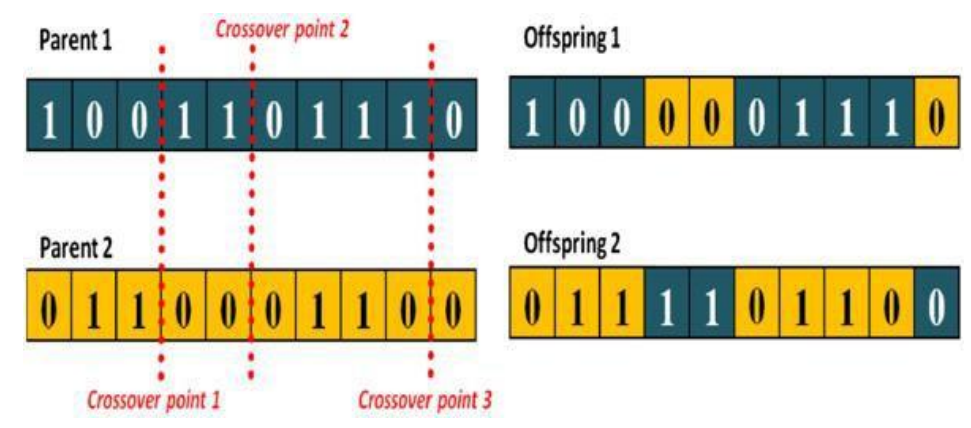

Figure 4. Crossover parent 1 and 2

Crossover system conducted in this research will be done in 10-crossover point where produce better result than 3-crossover points. After producing 2 binary result, where both results are decoded into a value and look for each fitness value. New fitness values namely Offspring 1 dan Offspring 2 will be compared with pareto front where the best value will be chosen as a solution.

The paper determined parameters: 1. "p" (the possibility of the message being sent back), which means the when the node population environtment occurs in a simulation the name of the broadcast will occur where a message can be sent many times where the message has arrived at the destination but the sender node sent messages continuously. 2. "Nr" is the permission given in the message to sent back. In this paper the value "Nr" depends on the number of node of population in a area, which the more number of population and the wider the area, the greater value of " $\mathrm{Nr}$ ". 3. "Dr" is the delay that occurs when sending the same message where the message has been sent 
does not arrive at the destination. 4. "TTL" where each message sent has a life time on protocol network, if the message has exceeded the specified time then the message will be lost so no flooding or bottlenecks occur in the protocol network.

\section{e. ICT Environment}

The Information and Communication Technology (ICT) infrastructure is quite important for the success of any online course. This means that adequate computers and internet connectivity ought to be avalaible to both learners and facilitators. The investigation revealed that 43 percent of the respondents (students) felt that the ICT infrastructure was not adequate. Thus, majority at 64 percent felt that facilitation to acquire own computer or tablet. Although the institution provided internet connectivity in the library, student computer laboratories and various hotspot whitin the institution, it seemed these were either not adequated or were not widely used for online learning and especially by students. This is because the each data message collected.

\section{f. Monitoring and Evaluation}

The online facilitation was normally monitored and evaluated on 3 month basis. Reports on various activities such as number discussion forums created, chat forums created, assignment given and marked, kind of quizzes given among other activities were reported. The reports were circulated to the departments concerned for follow up and feedback to the fasilitators concerned. These report were used to identify training gaps data for facilitators of learning courses by identifying commonly used and commonly neglected tools of the LMS.

\section{Research Results}

Analysis of protocol performance based on the amount of data dropped generally shown the higher maximum node speed the more data drops. In addition to the maximum speed of mobility, the pause time value also influences the amount of data dropped.

Network mobility is in accordance with the results of the test scenario in Table 1. The chart shows that throughput decrease due to an increase in the speed of node mobility. The chart shows that the delay decreases due to an increase in the speed of node mobility.

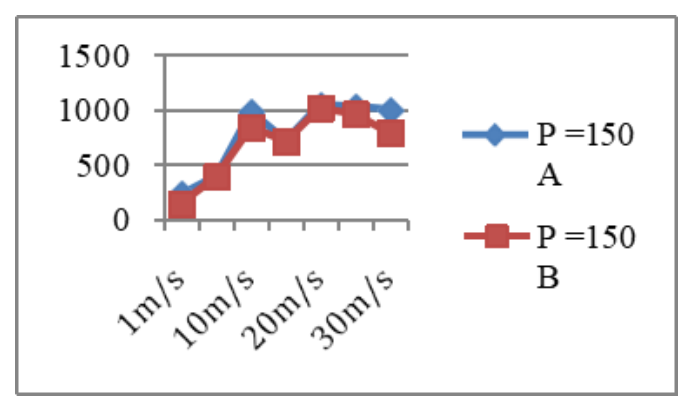

Figure 5. Chart Compare data drop

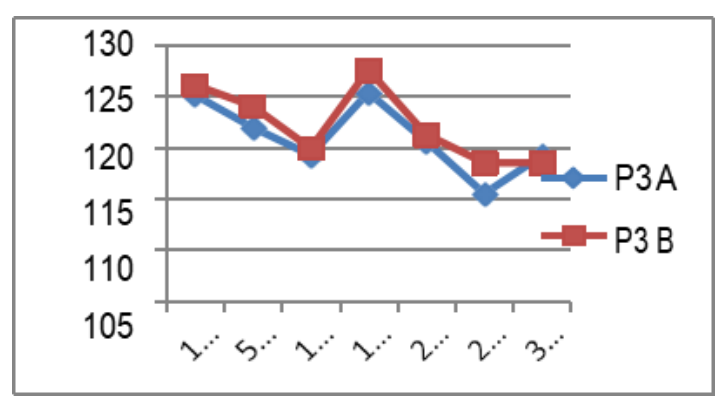

Figure 6. Chart Compare Throughput 


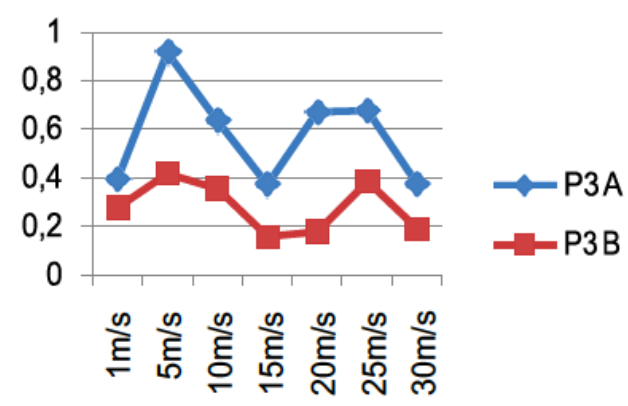

Figure 7. Chart based on Delay

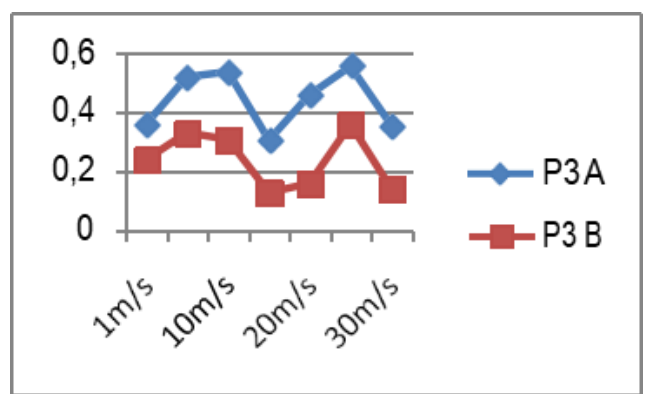

Figure 8. The Performance Delay Case using Optimized engine 200 node

After the analysis results are known about the researchers collaborating OLSR protocol, DSDV and Optimized engines that use EA, which in this study focuses on the problem of delay.

\section{Acknowledgment (if any)}

Although the OLSR routing protocol, DSDV has better performance than OLSR but the difference is still small or insignificant. This is because the mechanism for selecting stable routes is only based on the value of the route stability that has been obtained before. By NS2 and Optimized engine which uses the Evolutionary Algorithm will get the optimal configuration of the environment in accordance with the number of used node in population.and the elearning induction is normally carried out for whole month at beginning of a semester/session, not all student end up being trained during the training period. This situation was attribute to the fact that the induction is normally carried out at the beginning of semester when the learners are still undergoing orientation in the new environment and the author wanna say thank you to KEMENRISTEK LLDIKTI VII for the funding and Universitas Madura to our research site.

\section{References}

[1] W.Abdou., A.Henriet., \& Geethanjali, N.Journal of Network and Computer Applications, Elsevier.(2011)

[2] Johnson.Nadeem., M.Ullah., \& D.karim, Elsevier. (2011)

[3] Sharma., Manish., \& Singh, Gurpadam, (2011)

[4] W. Cordeiro., Aguiar, E. Moreira, W. Abelem, A. Stanton, M. Computer Communications and Networks. IEEE. (2007)

[5] P.Jacquet., P.Muhlethaler., \& T.Clausen. INRIA Rocquencourt, Rapport de recherche. (2001)

[6] T.Camp., J.Boleng., \& V. Davies, Wireless Communication \& Mobile Computing (WCMC), (pp. 483 502). (2002)

[7] D.Close., B.Robbins, A. D., Rubin, P. H., Stallman, R., \& Oostrum, v. P. The AWK Manual. Free Software Foundation, Inc. (1995) 
[8] A, Dana., A, Zadeh, K., \& Noori, S. A. 4th OLSR Interop / Work Shop. Ottawa, Canada. (2008)

[9] Jatmika, A. H.. ITS Surabaya. (2011)

[10] W. Su, , Lee, S.-J., \& Gerla, M. Mobility Prediction in Wireless Networks. IEEE Military Communications Conference, (pp. 491-495), (2000) 